\title{
Minimal Haemodynamic System Model Including Ventricular Interaction and Valve Dynamics
}

Bram W. Smith ${ }^{1}$, J. Geoffrey Chase ${ }^{2}$, Roger I. Nokes ${ }^{3}$, Geoffrey M.

Shaw $^{4}$ and Graeme Wake ${ }^{5}$

Department of Mechanical Engineering

University of Canterbury

Private Bag 4800

Christchurch

New Zealand

Email: g.chase@mech.canterbury.ac.nz

Fax: +64 364-2078

\footnotetext{
${ }^{1}$ Research Assistant

${ }^{2}$ Senior Lecturer

${ }^{3}$ Senior Lecturer, Dept of Civil Engineering, University of Canterbury

${ }^{4}$ Consultant, Christchurch Hospital Dept of Intensive Care

${ }^{5}$ Prof, Dept of Mathematics, University of Canterbury
} 


\begin{abstract}
Characterising circulatory dysfunction and choosing a suitable treatment is often difficult and time consuming, and can result in a deterioration in patient condition, or unsuitable therapy choices. A stable minimal model of the human cardiovascular system (CVS) is developed with the ultimate specific aim of assisting medical staff for rapid, on site modelling to assist in diagnosis and treatment. Models found in the literature simulate specific areas of the CVS with limited direct usefulness to medical staff. Others model the full CVS as a closed loop system, but models were found to be very complex, difficult to solve, or unstable. This paper develops a model that uses a minimal number of governing equations with the primary goal of accurately capturing trends in the CVS dynamics in a simple, easily solved, robust model. The model is shown to have long term stability and consistency with non-specific initial conditions as a result. An "open on pressure close on flow" valve law is created to capture the effects of inertia and the resulting dynamics of blood flow through the cardiac valves. An accurate, stable solution is performed using a method that varies the number of states in the model depending on the specific phase of the cardiac cycle, better matching the real physiological conditions. Examples of results include a $9 \%$ drop in cardiac output when increasing the thoracic pressure from $-4 \mathrm{mmHg}$ to $0 \mathrm{mmHg}$, and an increase in blood pressure from $120 / 80 \mathrm{mmHg}$ to $165 / 130 \mathrm{mmHg}$ when the systemic resistance is doubled. These results show that the model adequately provides appropriate magnitudes and trends that are in agreement with existing data for a variety of physiologically verified test cases simulating human CVS
\end{abstract}


function.

\section{Introduction}

Cardiovascular disease claims more lives than any other disease in westernised countries, affecting millions. Pin-pointing CVS dysfunction is often difficult because the clinical signs, or the availability and interpretation of physiological measurements are unreliable. Often patient specific information is incomplete and/or confusing as it comes from a diverse range of sources such as invasive and non-invasive pressure measurements, flow rates and ECG signals. Health professionals therefore rely on intuition and experience to make a 'clinical' diagnosis and treatment decisions. Sometimes this approach results in multiple therapies being applied until a suitable treatment is found. Poor outcomes result from failure to quickly and correctly diagnose and treat the underlying condition.

This research develops a minimal model of the cardiovascular system to assist health professionals in the key areas of understanding, diagnosis and therapy selection. An appropriate CVS model can identify inconsistencies and irregularities in patient measurements, to help choose suitable fluid, drug, or mechanical interventions using a patient specific model [Frazier et al., 2001; Westaby et al., 2000].

Most current approaches to modelling the CVS can be grouped into either Finite Element (FE) or Pressure Volume (PV) approaches. FE techniques offer microscale results that can theoretically be very accurate both in magnitude and in trends. To gain such accuracy requires immensely detailed 
inputs such as muscle fibre orientations, structures and mechanical properties [Peskin and McQueen, 1992; McQueen et al., 1982; Legrice et al., 1997; Smaill and Hunter, 1991]. Not only can such patient specific information be difficult to obtain, but the computational power required is too extreme for clinical use. FE methods are good for assisting in understanding of general heart function, but they are not suitable for patient specific rapid diagnostic feedback.

PV methods divide the CVS system into a series of elastic chambers separated by resistances, and inertial effects where required. Each elastic chamber models a section of the CVS such as the ventricles, the atria, or the aorta, each with their own pressure-volume relationship. Only a minimal number of parameters, such as chamber elastances and arterial resistances, are required. These models can be solved on desktop computers in times suitable for immediate feedback. While there are many examples of PV, lumped parameter approaches in the literature, most are focused on simulating only certain areas of CVS function [Amoore et al., 1992; Beyar et al., 1987; Burkhoff and Tyberg, 1993; Chung et al., 1997; Ursino, 1999; Stergiopulos et al., 1999].

PV lumped parameter models in the literature generally work in similar ways. Amoore et.al. (1992) focuses primarily on the dynamics of ventricular interaction, with little reference to the layout or dynamics of the remaining CVS. Closed loop models in Burkhoff et.al. (1993) and Beyar et.al. (1993) outline in more detail the CVS layout, but have minimal consideration for inertia. Burkhoff et.al. does not include inertial effects, while Beyar et.al. uses inertia in all equations, including areas of the CVS where there is minimal fluctuation in velocity. Other models were found to lack robustness. For 
example, simulations by this author using the model of Chung et.al. (1996) found no steady state solution after long simulation times due to over defined governing equations.

Hence, the model developed must fulfil the following goals to be suitable for rapid diagnostic feedback:

- Model parameters can be relatively easily determined or approximated for a specific patient.

- The model can be run on a desktop computer in reasonable time.

- Accurate prediction of trends.

- The full closed-loop model must be stable with minimal complexity and physiologically realistic inertia and valve effects.

These goals are set to restrict the model from becoming too complex while ensuring it's practicality. The limitations on the patient specific information, computational power and time mean the PV modelling method offers the greatest potential for fulfilling these requirements. A "Minimal Model" approach to CVS modelling means using a minimal number of governing equations and parameters where other similar models in the literature use many variables and complex formula.

\section{Method}

Ultimately, the model presented is intended to simulate the essential haemodynamics of the cardiovascular system including the heart, and the pulmonary and systemic circulation systems. The full model presented is shown 
in Figure 1, with two ventricles and additional chambers for the systemic and pulmonary circulations. The atria have not been added as they contribute only minimally to main cardiac trends and can be easily added for specific cases. The ventricles are coupled to account for important ventricular interaction dynamics.

In Figure 1 resistances, labelled $\mathrm{R}$, simulate the resistance experienced by the blood passing through the arteries or valves between elastic chambers. Inductors, labelled L, show where the effects of inertia are included. Inertial effects are only included where the blood goes through large changes in velocity, such as around the ventricle exit valves [Melchior et al., 1992]. Diodes show the one-way valves at the inlet and exit of the ventricles.

\subsection{The Cardiac Chamber}

A single active cardiac chamber is first developed to understand the dynamics of the ventricles, and is similar to Windkessel circuits in the literature, but with an elastic chamber rather than a capacitor [Tsitlik et al., 1992; Santamore and Burkhoff, 1991].

\section{The PV Diagram and Relationship}

PV diagrams for elastic chambers, as schematically shown in Figure 2, are extensively used to analyse ventricle pumping mechanics. Two main characteristics of the PV diagram are the End Systolic Pressure-Volume Relationship (ESPVR) and the End Diastolic Pressure-Volume Relationship (EDPVR), which define the upper and lower limits of the cardiac cycle. 
Equations approximating the ESPVR and EDPVR lines are widespread [Hardy and Collins, 1982; Maughan et al., 1987; Hunter et al., 1983; Chung et al., 1997; Santamore and Burkhoff, 1991; Beyar et al., 1987; Amoore et al., 1992]. The most commonly used relationships are defined [Chung et al., 1997; Santamore and Burkhoff, 1991; Beyar et al., 1987]:

$$
\begin{gathered}
\mathrm{P}_{\mathrm{es}}(\mathrm{V})=\mathrm{E}_{\mathrm{es}}\left(\mathrm{V}-\mathrm{V}_{\mathrm{d}}\right) \\
\mathrm{P}_{\text {ed }}(\mathrm{V})=\mathrm{A}\left(\mathrm{e}^{\lambda\left(\mathrm{V}-\mathrm{V}_{\mathrm{o}}\right)}-1\right)
\end{gathered}
$$

where Equation (1) is the linear relationship between the end systolic pressure $\left(\mathrm{P}_{\mathrm{es}}\right)$ and volume $(\mathrm{V})$ with elastance $\left(\mathrm{E}_{\mathrm{es}}\right)$ and the volume at zero pressure $\left(V_{d}\right)$. Equation (2) defines the non-linear relationship between end diastolic pressure $\left(\mathrm{P}_{\mathrm{ed}}\right)$ and volume $(\mathrm{V})$ with the parameters $\mathrm{A}, \lambda$, and $\mathrm{V}_{0}$.

\section{Cardiac Driving Function}

Time varying elastance $(\mathrm{E}(\mathrm{t}))$ is used to model cardiac muscle activation [Beyar et al., 1987; Chung et al., 1997; Burkhoff and Tyberg, 1993; Santamore and Burkhoff, 1991]. The upper and lower limits of the elastance are defined in Figure 2, as the slopes of the ESPVR and EDPVR lines [Chung, 1996]. A function e(t) shown in Figure 3, represents the variance of elastance between minimum and maximum values over a single heart beat. Combining the driver definition with Equations (1) and (2) produces an equation for the pressure volume relationship in a single chamber in terms of chamber pressure $\left(P_{2}\right)$, volume $(\mathrm{V})$ and time $(\mathrm{t})$. 


$$
\begin{gathered}
\mathrm{P}_{2}(\mathrm{~V}, \mathrm{t})=\mathrm{e}(\mathrm{t}) \mathrm{P}_{\mathrm{es}}(\mathrm{V})+(1-\mathrm{e}(\mathrm{t})) \mathrm{P}_{\text {ed }}(\mathrm{V}) \quad 0 \leq \mathrm{e}(\mathrm{t}) \leq 1 \\
\mathrm{P}_{2}(\mathrm{~V}, \mathrm{t})=\mathrm{e}(\mathrm{t}) \mathrm{E}_{\mathrm{es}}\left(\mathrm{V}-\mathrm{V}_{\mathrm{d}}\right)+(1-\mathrm{e}(\mathrm{t})) \mathrm{A}\left(\mathrm{e}^{\lambda\left(\mathrm{V}-\mathrm{V}_{\mathrm{o}}\right)}-1\right) \\
\mathrm{e}(\mathrm{t})=\sum_{\mathrm{i}=1}^{\mathrm{N}} \mathrm{A}_{\mathrm{i}} \mathrm{e}^{-\mathrm{B}_{\mathrm{i}}\left(\mathrm{t}-\mathrm{C}_{\mathrm{i}}\right)^{2}}
\end{gathered}
$$

Equation (4) governs the time dependent pressure volume relationship in an active elastic chamber or ventricle. Equation (5) defines the driver function where $A_{i}, B_{i}, C_{i}$ and $N$ determine the shape of the driver profile. To produce the profile in Figure $3, \mathrm{~A}=1, \mathrm{~B}=80, \mathrm{C}=0.27$ and $\mathrm{N}=1$ were used. Chung et.al. (1996) uses a more complex driver, while many other variations can be found in the literature with very similar shapes [Amoore et al., 1992; Beyar et al., 1987; Burkhoff and Tyberg, 1993; Senzaki et al., 1996]. The profile of this driver function $(\mathrm{e}(\mathrm{t}))$ can be compared with experimentally determined variations in ventricular elastance [Segers et al., 2000a,b; Senzaki et al., 1996]. However, the simple profile presented was chosen to limit it's contribution to model dynamics, enabling focus on the mechanical function of the model.

\section{Fluid Flow Rate Definitions}

The governing equations for fluid flow through the arteries is dependent on whether inertial effects are included. If inertia is ignored or negligible, the flow rate can be derived from electrical circuit analogies and result in Poiseuille's equation [Fung, 1990; Beyar et al., 1987; Chung, 1996; Olansen et al., 2000]. 


$$
\mathrm{Q}_{1}=\frac{\mathrm{P}_{1}-\mathrm{P}_{2}}{\mathrm{R}_{1}}
$$

where the resistance $(\mathrm{R})$ is defined by the Poiseuille's equation as $\mathrm{R}=8 \mu \mathrm{l} / \pi r_{0}^{4}$, for radius, $\mathrm{r}_{0}$, fluid viscosity, $\mu$, and artery length, $\mathrm{l}$.

When applying Equation (6), all the assumptions associated with the Poiseuille equation apply, including rigid walls and fully developed laminar flow. Equation (6) allows discontinuous changes in flow rate, ignoring inertial effects. When inertial effects are added, the equation of motion for the flow becomes a first order differential equation for flow rate that may also be derived from electrical circuit theory [Beyar et al., 1987; Hoppensteadt and Peskin, 2002].

$$
\frac{\mathrm{dQ}}{\mathrm{dt}}=\frac{\mathrm{P}_{1}-\mathrm{P}_{2}-\mathrm{Q}_{1} \mathrm{R}_{1}}{\mathrm{~L}_{1}}
$$

Equations (6) and (7) assume constant resistance, so resistance does not vary with flow rate as a result of the fully developed flow assumption. This model only includes inertial effects where the velocity fluctuations are significant, such as in the aorta and the pulmonary artery. Elsewhere, Equation (6) is used. Therefore, the rate of change of chamber volume is governed by the net flow rate:

$$
\dot{\mathrm{V}}=\mathrm{Q}_{\text {in }}-\mathrm{Q}_{\text {out }}
$$

To summarize, for any given volume $(\mathrm{V})$ and time $(\mathrm{t})$, the pressure in the chamber $\left(\mathrm{P}_{2}(\mathrm{~V}, \mathrm{t})\right)$ can be calculated using Equation (4). The flow rates are 
then calculated using either Equations (6) or (7), and from the flow rate, the rate of change of volume is calculated using Equation (8). These equations define the dynamics of a single ventricle model with resistances at the inlet and outlet.

\subsection{Ventricular Interaction}

Direct ventricular interaction has a significant impact on cardiovascular dynamics, and is caused by both the septum and the pericardium. The septum is an active flexible common wall between the left and right ventricle, and the pericardium is a relatively rigid, passive wall that encapsulates the entire heart. The double lines between the left and right ventricles in Figure 1 indicate the coupling due to ventricular interaction. Figure 4 and Table 1 define the variables and cardiac geometry used in the following definitions for ventricular interaction.

\section{Volumes}

Figure 4 shows the left and right ventricle volumes and the three different free walls of the ventricles and the septum. The free wall volumes, $V_{\text {lvf }}, V_{\text {rvf }}$ and $\mathrm{V}_{\mathrm{spt}}$, are not physical volumes, but are defined to capture the deflection of the cardiac free walls relative to the ventricle volumes. The left ventricle free wall volume $\left(\mathrm{V}_{\mathrm{lvf}}\right)$, using Figure 4 is defined:

$$
\mathrm{V}_{\mathrm{lvf}}=\mathrm{V}_{\mathrm{lv}}-\mathrm{V}_{\mathrm{spt}}
$$

Similarly, the right ventricle free wall volume $\left(\mathrm{V}_{\mathrm{rvf}}\right)$ is defined: 


$$
\mathrm{V}_{\mathrm{rvf}}=\mathrm{V}_{\mathrm{rv}}+\mathrm{V}_{\mathrm{spt}}
$$

Finally, the pericardium volume $\left(\mathrm{V}_{\mathrm{pcd}}\right)$ is defined:

$$
\mathrm{V}_{\mathrm{pcd}}=\mathrm{V}_{\mathrm{lv}}+\mathrm{V}_{\mathrm{rv}}=\mathrm{V}_{\mathrm{lvf}}+\mathrm{V}_{\mathrm{rvf}}
$$

The total volume of the pericardium defined excludes the volume of the atria and the myocardium, although the model may be readily augmented to include these details.

\section{Pressures}

Given the volumes of the cardiac chambers, the governing PV relationships, as defined in Equation (4), can be used to calculate the pressures. The specific pressure relations are defined using Figure 4 for the left and right ventricle, and the pericardium using the variables in Table 1.

$$
\begin{gathered}
\mathrm{P}_{\mathrm{lv}}=\mathrm{P}_{\mathrm{lvf}}+\mathrm{P}_{\text {peri }} \\
\mathrm{P}_{\mathrm{rv}}=\mathrm{P}_{\mathrm{rvf}}+\mathrm{P}_{\text {peri }} \\
\mathrm{P}_{\text {peri }}=\mathrm{P}_{\text {pcd }}+\mathrm{P}_{\text {th }}
\end{gathered}
$$

The septum pressure, using Equations (12) and (13), is therefore defined:

$$
\mathrm{P}_{\mathrm{spt}}=\mathrm{P}_{\mathrm{lv}}-\mathrm{P}_{\mathrm{rv}}=\mathrm{P}_{\mathrm{lvf}}-\mathrm{P}_{\mathrm{rvf}}
$$


The left and right ventricle and septal free wall pressures are a function of the driver, $\mathrm{e}(\mathrm{t})$, and the end systolic and end diastolic pressures, $\mathrm{P}_{\text {ed }}$ and $\mathrm{P}_{\mathrm{es}}$ respectively, in the same form as Equations (3) and (4).

$$
\begin{gathered}
\mathrm{P}_{\text {lvf }}=e \mathrm{P}_{\mathrm{es}, \mathrm{lvf}}+(1-\mathrm{e}) \mathrm{P}_{\mathrm{ed}, \mathrm{lvf}} \quad \mathrm{P}_{\mathrm{rvf}}=\mathrm{e} \mathrm{P}_{\mathrm{es}, \mathrm{rvf}}+(1-\mathrm{e}) \mathrm{P}_{\mathrm{ed}, \mathrm{rvf}} \\
\mathrm{P}_{\mathrm{spt}}=\mathrm{eP}_{\mathrm{es}, \mathrm{spt}}+(1-\mathrm{e}) \mathrm{P}_{\mathrm{ed}, \mathrm{spt}}
\end{gathered}
$$

Finally, the pressure across the pericardium wall is defined by the following non-linear PV relationship [Chung et al., 1997]:

$$
\mathrm{P}_{\mathrm{pcd}}\left(\mathrm{V}_{\mathrm{pcd}}\right)=\mathrm{P}_{0, \mathrm{pcd}}\left(\mathrm{e}^{\lambda_{\mathrm{pcd}}\left(\mathrm{V}_{\mathrm{pcd}}-\mathrm{V}_{0, \mathrm{pcd}}\right)}-1\right)
$$

Equations (12)-(18) are employed to obtain the pressure in the left and right ventricles. Once the ventricle pressures are known the flow rates can be determined and the rate of change of volume calculated.

\subsection{The Peripheral Circulation}

To capture more of the major governing haemodynamics, the circuit must be closed so that fluid can flow around the entire loop, as in Figure 1. The output of each cardiac chamber is connected to the inlet of the other cardiac chamber by the pulmonary and systemic circulations. These peripheral circulation systems are modelled using elastic chambers, simulating the arteries and veins, and resistances, simulating blood flow through the capillaries. 
The dynamics of the cardiac chambers will now be affected by peripheral elastances and resistances, capturing the essential dynamics of the pulmonary and systemic circulations.

Although the non-linear PV relation defined in Equation (2) may be more suitable to define peripheral elastances, it requires knowledge of $\mathrm{A}, \lambda$, and $\mathrm{V}_{0}$. To minimise complexity, the model employs the linear PV relationship in Equation (1), requiring only $\mathrm{E}_{\mathrm{es}}$ and $\mathrm{V}_{\mathrm{d}}$. The effects of inertia are not included in the peripheral resistances, $\left(\mathrm{R}_{\text {sys }}\right.$ and $\left.\mathrm{R}_{\text {pul }}\right)$ and the systemic and pulmonary veins $\left(R_{\mathrm{pu}}\right.$ and $\left.\mathrm{R}_{\mathrm{vc}}\right)$ because changes in blood flow velocity are damped in the arteries and become negligible in the body and lungs.

\subsection{Dynamic System Models and Simulation}

\section{Single Chamber}

For the single active cardiac chamber model with no inertia, the minimal model is governed by Equation (8), requiring only the volume as a state variable $(\underline{\mathbf{x}}=[\mathrm{V}])$. With the incorporation of inertial effects the flow (Q) is governed by Equation (7) and both inflow and outflow rates become state variables $\left(\underline{\mathbf{x}}=\left[\begin{array}{lll}\mathrm{V} & \mathrm{Q}_{1} & \mathrm{Q}_{2}\end{array}\right]\right)$. Thus, the nonlinear state derivative $(\underline{\dot{x}})$ for a 
single chamber with inertia is defined:

$$
\underline{\dot{\mathrm{x}}}=\left[\begin{array}{c}
\dot{\mathrm{V}} \\
\dot{\mathrm{Q}_{1}} \\
\dot{\mathrm{Q}}_{2}
\end{array}\right]=\left[\begin{array}{r}
\mathrm{Q}_{1}-\mathrm{Q}_{2} \\
\frac{\mathrm{P}_{1}-\mathrm{P}_{2}-\mathrm{Q}_{1} \mathrm{R}_{1}}{\mathrm{~L}_{1}} \\
\frac{\mathrm{P}_{2}-\mathrm{P}_{3}-\mathrm{Q}_{2} \mathrm{R}_{2}}{\mathrm{~L}_{2}}
\end{array}\right]
$$

\section{Valve Law}

In models where inertia is not included and the governing flow is defined by Equation (6), the valves are simulated by setting the flow rate to zero when it is calculated to be zero or negative. When inertial effects are included, the flow rate (Q) is governed by the first order ODE of Equation (7). Holding the flow rate at zero will now create a discontinuity and an unstable numerical solution. Of the numerous models in the literature, few mentioned valve function, and the author could not find a realistic valve law for models that use inertia. The solution developed allows the system state to change dynamically while solving.

Figure 2 shows the three different states that a single cardiac chamber cycles through each heartbeat. During ejection and filling, the state vector consists of the volume and a flow rate out or in respectively. During isovolumetric contraction and relaxation, only the volume (V) is required. Hence, different parts of the cardiac cycle require different state variables.

To change the state of the system, the state variables are triggered to change when a flow rate, or a pressure gradient, becomes negative. When a flow rate $(\mathrm{Q})$ becomes negative, it is removed from the state vector to ac- 
count for the valve closing in the absence of flow. When a pressure gradient becomes negative, favouring forward flow, the associated flow rate is reincluded in the state vector to account for the valve opening. Hence, the valve law for the model presented is "close on flow, open on pressure" where the valve opens on a negative pressure gradient, but is delayed from closing on a positive pressure gradient due to the inertia of the blood, matching known physiological response [Opie, 1998].

\section{Ventricular Interaction}

When simulating ventricular interaction, the dynamics of the septum and the effects of the pericardium must be taken into account. The following steps outline the sequence for determining the state derivative at each time step:

1. State variables $V_{\text {lv }}$ and $V_{r v}$ are obtained at each time step.

2. Equation (14) is used to find $\mathrm{P}_{\text {peri }}$ from Equation (18) and Equation (11)

3. Substituting Equation (16) and Equation (17) into $\mathrm{P}_{\mathrm{spt}}=\mathrm{P}_{\mathrm{lvf}}-\mathrm{P}_{\mathrm{rvf}}$ relates $\mathrm{V}_{\mathrm{lv}}$ and $\mathrm{V}_{\mathrm{rv}}$ to $\mathrm{V}_{\mathrm{spt}}$ :

$$
\begin{aligned}
& e E_{e s, s p t}\left(V_{s p t}-V_{d, s p t}\right)+(1-e) P_{o, s p t}\left(e^{\lambda_{s p t}\left(V_{s p t}-V_{o, s p t}\right)}-1\right) \\
& =e E_{e s, l v f}\left(V_{l v}-V_{s p t}-V_{d, l v f}\right)+(1-e) P_{o, l v f}\left(e^{\lambda_{l v f}\left(V_{l v}-V_{s p t}-V_{o, l v f}\right)}-1\right) \\
& -e E_{e s, r v f}\left(V_{r v}+V_{s p t}-V_{d, r v f}\right)-(1-e) P_{o, r v f}\left(e^{\lambda_{r v f}\left(V_{r v}+V_{s p t}-V_{o, r v f}\right)}-1\right)
\end{aligned}
$$


where the subscript denotes which cardiac free wall the properties apply to. Equation (20) can be used to determine $V_{\text {spt }}$ given $V_{\text {lv }}$ and $V_{\text {rv }}$ via zero finding solutions.

4. Given $\mathrm{V}_{\text {spt }}$ and Equations (9) and (10), $\mathrm{V}_{\mathrm{lvf}}$ and $\mathrm{V}_{\mathrm{rvf}}$ can be determined and used in Equation (16) to calculate $\mathrm{P}_{\mathrm{lvf}}$ and $\mathrm{P}_{\mathrm{rvf}}$.

5. The values of $\mathrm{P}_{\text {lvf }}, \mathrm{P}_{\mathrm{rvf}}$ and $\mathrm{P}_{\text {peri }}$ are used in Equations (12) and (13) to find $\mathrm{P}_{\mathrm{lv}}$ and $\mathrm{P}_{\mathrm{rv}}$.

6. The ventricle pressures are then used to calculate the flow rates, and thus, the rate of change of ventricle volumes $\left(\mathrm{dV}_{\mathrm{lv}} / \mathrm{dt}\right.$ and $\left.\mathrm{dV} \mathrm{rv} / \mathrm{dt}\right)$.

These six steps are repeated at each time step. The initial volumes are approximated based on normal heart function and the initial flow rates are calculated using Poiseuille's equation as given in Equation (6).

\section{Results}

Models were simulated with increasing complexity starting with a single chamber model with no inertia. Inertia was added next, followed by ventricular interaction, and finally, a full closed loop model of the heart and circulation.

Figure 5 plots variations in chamber pressure and volume for a single cardiac chamber with constant inlet and outlet pressures. The plot shows the model rapidly settling to the same steady state solution within two heart beats from two very different initial conditions. The rate of convergence from different initial conditions highlights the robustness and stability of this 
approach . Simulations were also run with inertial effects included where the inertance $(\mathrm{L})$ has been set to a very small value $\left(\mathrm{L}=10^{-6} \mathrm{mmHgs}^{2} / \mathrm{ml}\right)$. This model produced results that exactly match those without inertial effects, as expected.

The next model simulated ventricular interaction with constant inlet and outlet pressures. Figure 6 shows the movement of the septum during the cardiac cycle. The septum volume oscillates over a magnitude of $3 \mathrm{ml}$, or about $4 \%$ of the ventricle volume, during the cardiac cycle matching published results [Chung et al., 1997].

Figure 7 shows the output pressures and volumes for the closed loop model shown in Figure 1. The patient specific parameters are extrapolated from those found over several references, producing model results comparable to an average human [Burkhoff and Tyberg, 1993; Chung, 1996; Ursino, 1999]. A fixed heart rate of 80 beats/minute, and a total stressed blood volume of 1500 milli-litres are used to match normal human parameters [Parsons, 2002].

The target performance metrics of the full closed-loop model were taken from Guyton (1991). A typical ventricle stroke volume (SV) is about $70 \mathrm{ml}$ as marked in Figure 7. An average person's aortic pressure is about 120/80 $\mathrm{mmHg}$ as marked (ao), while a normal pulmonary artery pressure is approximately $25 / 8 \mathrm{mmHg}$ as marked (pa). The return pressure entering the right heart $\left(\mathrm{P}_{\mathrm{vc}}\right)$ is typically around $0 \mathrm{mmHg}$, but can be negative since the average pressure in the thoracic cavity is generally about $-4 \mathrm{mmHg}$. The return pressure to the left ventricle $\left(\mathrm{P}_{\mathrm{pv}}\right)$ is typically $1-5 \mathrm{mmHg}$. All of these magnitudes are comparable to values found in standard medical references, 
verifying the model structure and assumptions, and the solution method.

Since the ultimate goal is simulation of human heart function in response to changes in therapy, tests to validate model trends were carried out for known physiological trends. For example, if $\mathrm{P}_{\text {th }}$ is increased, as occurs during positive pressure mechanical ventilation, cardiac output $(\mathrm{CO})$ is decreased [Guyton, 1991]. Figure 8 shows results for a normal $(-4 \mathrm{mmHg})$ and an increased $(0 \mathrm{mmHg})$ thoracic pressure where the stroke volume, and thus the cardiac output, is decreased by $9 \%$, which is in good agreement with clinical data for this intervention.

A second test shows the effect of changing the systemic circulatory resistance. Clinical data shows that an acute increase in resistance results in reduced cardiac output that varies with the magnitude of the change. Figure 9 shows that as systemic resistance decreases, stroke volume increases, meaning an increase in cardiac output. The opposite trend occurs as the resistance is increased. Figure 10 shows the rise in pressure in the aorta as a result of increased peripheral resistance, matching the increase in blood pressure in patients with narrowed or blocked arteries. Hence, the model captures known clinical behaviour in response to changes in systemic resistance.

Figure 11 shows the effect of varying ventricle contractility, a measure of cardiac pump function [Maughan and Kass, 1988]. Contractility is varied in the model by changing the end systolic elastance, $\mathrm{E}_{\mathrm{es}}$ [Burkhoff and Tyberg, 1993]. Typically, increased contractility increases cardiac output and this trend is shown in Figure 11. 


\section{Discussion}

The results show the minimal model presented captures major physiological trends while avoiding instability and robustness problems found in other published models. More complex models such as that developed by Chung et.al. (1996) were found to be very dependent on accurate calculation of specific initial conditions. That model converged slowly because it had too many governing equations, over-defining the model. The minimal model presented avoids these numerical instability and inconsistency problems by using only a minimal number of variables.

Research by Michard and Teboul (2002) notes another application for this model in predicting fluid responsiveness in ICU patients. They concluded that static indicators of cardiac preload, such as pressures and volumes in the heart, poorly predicted response to fluid therapies. However, dynamic parameters such as variations in blood pressures during respiration are significantly higher in those patients who respond well to fluid therapies. The minimal model presented readily captures these subtle variations in cardiac dynamics that may not be seen in standard ICU measurements.

The good agreement of the results with clinical data shows the potential of this model for predicting trends in the CVS. With future development of the model, more subtle trends in the CVS haemodynamics can be simulated. In addition, effective methods for identifying patient specific parameters will be an important part of future development. 


\section{Conclusions}

The advantages in numerical stability and model robustness of taking the minimal model approach to CVS modelling are presented. The presented model combines a simple closed-loop model, using minimal variables, with inertial effects and a realistic valve law. The individual elements of the model are first simulated independently to gain understanding of their independent function. An open on pressure, close on flow, valve function consistent with normal physiological valve function is presented. The results were found to be stable, consistent and in good agreement with known clinical data for normal human heart function. More specifically, the complete closed loop system model was able to accurately capture trends related to increases in thoracic pressure, systemic resistance, and cardiac contractility, as well as the dynamics of septal interaction. The overall results show the potential of modelling the cardiovascular system in this fashion, and ultimately, in becoming an effective aid for medical professionals in the diagnosis and treatment of CVS dysfunction.

\section{References}

Amoore, J., Santamore, W., Corin, W., and George, D. (1992). Computer simulation of the effects of ventricular interdependence on indices of left ventrilcular systolic function. J. Biomed. Eng, 14:257-262. [P-V relations].

Beyar, R., Hausknecht, M. J., Halperin, H. R., Yin, F. C., and Weisfeldt, 
M. L. (1987). Interaction between cardiac chambers and thoracic pressure in intact circulation. Am. J. Physiol., 22(253):H1240-H1252.

Burkhoff, D. and Tyberg, J. V. (1993). Why does pulmonary venous pressure rise after onset of lv dysfunction: a theoretical analysis. Am. J. Physiol., 265:H1819-H1828.

Chung, D. (1996). Ventricular Interaction in a Closed-Loop model of the canine circulation. Master of science, Rice University.

Chung, D., Niranjan, S., Clark, J., Bidani, A., and Johnston, W. (1997). A dynamic model of ventricular interaction and pericardial influence. Am. J. Physiol., 14(272):H2924-H2962.

Frazier, O. H., Myers, T. J., Jarvik, R. K., Westaby, S., Pigott, D. W., Gregoric, I. D., Khan, T., Tamez, D. W., Conger, J. L., and Macris, M. P. (2001). Research and development of an implantable, axial-flow left ventricular assist device: the jarvik 2000 heart. Ann Thorac Surg, 71(3 Suppl):S125-32; discussion S144-6.

Fung, Y. (1990). Biomechanics: Motion, flow, stress, and growth. SpringerVerlag, San Deigo.

Guyton, A. C. (1991). Textbook of medical physiology. W.B. Saunders Company, Philadelphia, 8 edition.

Hardy, H. H. and Collins, R. E. (1982). On the pressure-volume relationship in circulatory elements. Med Biol Eng Comput, 20(5):565-70. 
Hoppensteadt, F. C. and Peskin, C. S. (2002). Modeling and Simulation in Medicine and the Life Sciences. Springer-Verlag, New York.

Hunter, W. C., Janicki, J. S., Weber, K. T., and Noordergraff, A. (1983). Systolic mechnical properties of the left ventricle. Circulation Research, 52(3):319-327.

Legrice, I., Hunter, P., and Smaill, B. (1997). Laminar structure of the heart: a mathematical model. Am J Physiol, 272:H2466-76.

Maughan, W. and Kass, D. A. (1988). The use of the pressure-volume diagram for measuring ventricular pump function. Automedica, 11:317-342.

Maughan, W. L., Sunagawa, K., and Sagawa, K. (1987). Ventricular systolic interdependence: volume elastance model in isolated canine hearts. Am. J. Physiol., 253:H1381-H1390. [Ventricular interaction].

McQueen, D. M., Peskin, C. S., and Yellin, E. L. (1982). Fluid dynamics of the mitral valve: physiological aspects of a mathematical model. Am J Physiol, 242(6):H1095-110.

Melchior, F. M., Sriniwasan, R. S., and Charles, J. B. (1992). Mathematical modeling of human cardiovascular system for simulation of orthostatic response. Am. J. Physiol., 262:H1920-H1933.

Michard, F. and Teboul, J. L. (2002). Predicting fluid responsiveness in icu patients: a critical analysis of the evidence. Chest, 121(6):2000-8.

Olansen, J., Clark, J., Khoury, D., Ghorbel, F., and Bidani, A. (2000). A 
closed-loop model of the canine cardiovascular system that includes ventricular interaction. Comp. Biomed. Res., 33:260-295.

Opie, L. H. (1998). The heart : physiology, from cell to circulation, 3rd ed. Lippincott-Raven, Philadelphia.

Parsons, R. H. (2002). Human parameters. http://bio.bio.rpi.edu/Parsons /Universal/2HemorVolume\%20Lab /HemPar2.html.

Peskin, C. S. and McQueen, D. M. (1992). Cardiac fluid dynamics. Crit Rev Biomed Eng, 20(5-6):451-9.

Santamore, W. P. and Burkhoff, D. (1991). Hemodynamic consequences of ventricular interaction as assessed by model analysis. Am. J. Physiol., 260:H146-H157.

Segers, P., Stergiopulos, N., Schreuder, J. J., Westerhof, B. E., and Westerhof, N. (2000a). Left ventricular wall stress normalization in chronic pressure-overloaded heart: a mathematical model study. Am J Physiol Heart Circ Physiol, 279(3):H1120-7.

Segers, P., Stergiopulos, N., and Westerhof, N. (2000b). Quantification of the contribution of cardiac and arterial remodeling to hypertension. $H y$ pertension, 36(5):760-5.

Senzaki, H., Chen, C. H., and Kass, D. A. (1996). Single-beat estimation of end-systolic pressure-volume relation in humans. a new method with the potential for noninvasive application. Circulation, 94(10):2497-506. 
Smaill, B. and Hunter, P. (1991). Structure and function of the diastolic heart: Material properties of passive myocardium. In Glass, L., Hunter, P., and McCulloch, A., editors, Theory of Heart, pages 1-29. SpringerVerlag, Harrisonburg.

Stergiopulos, N., Segers, P., and Westerhof, N. (1999). Use of pulse pressure method for estimating total arterial compliance in vivo. Am J Physiol, 276(2 Pt 2):H424-8.

Tsitlik, J. E., Halperin, H. R., Popel, A. S., Shoukas, A. A., Yin, F. C., and Westerhof, N. (1992). Modeling the circulation with three-terminal electrical networks containing special nonlinear capacitors. Ann Biomed Eng, 20(6):595-616.

Ursino, M. (1999). A mathematical model of the carotid baroregulation in pulsating conditions. IEEE Trans Biomed Eng, 46(4):382-92.

Westaby, S., Banning, A. P., Jarvik, R., Frazier, O. H., Pigott, D. W., Jin, X. Y., Catarino, P. A., Saito, S., Robson, D., Freeland, A., Myers, T. J., and Poole-Wilson, P. A. (2000). First permanent implant of the jarvik 2000 heart. Lancet, 356(9233):900-3. 


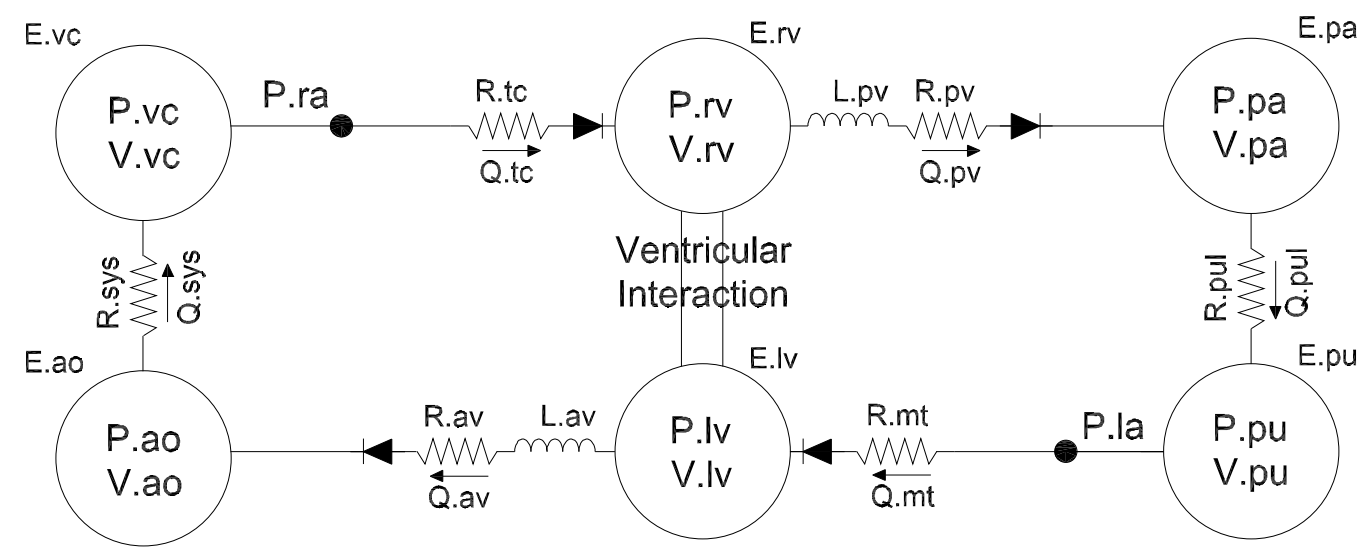

Figure 1: The presented closed loop model of the cardiovascular system. 


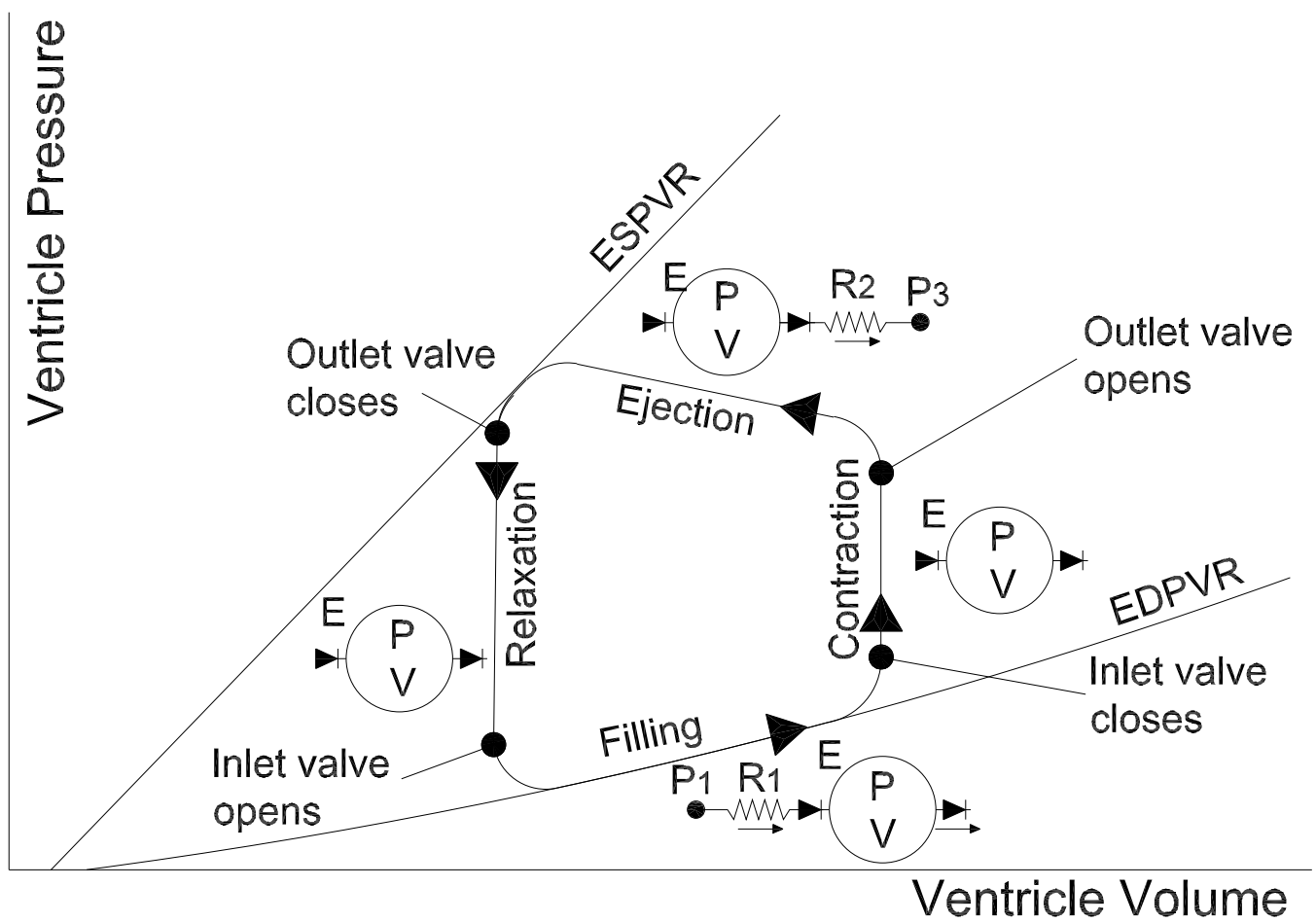

Figure 2: An example of a pressure volume diagram with the different states of the cardiac chamber included. 


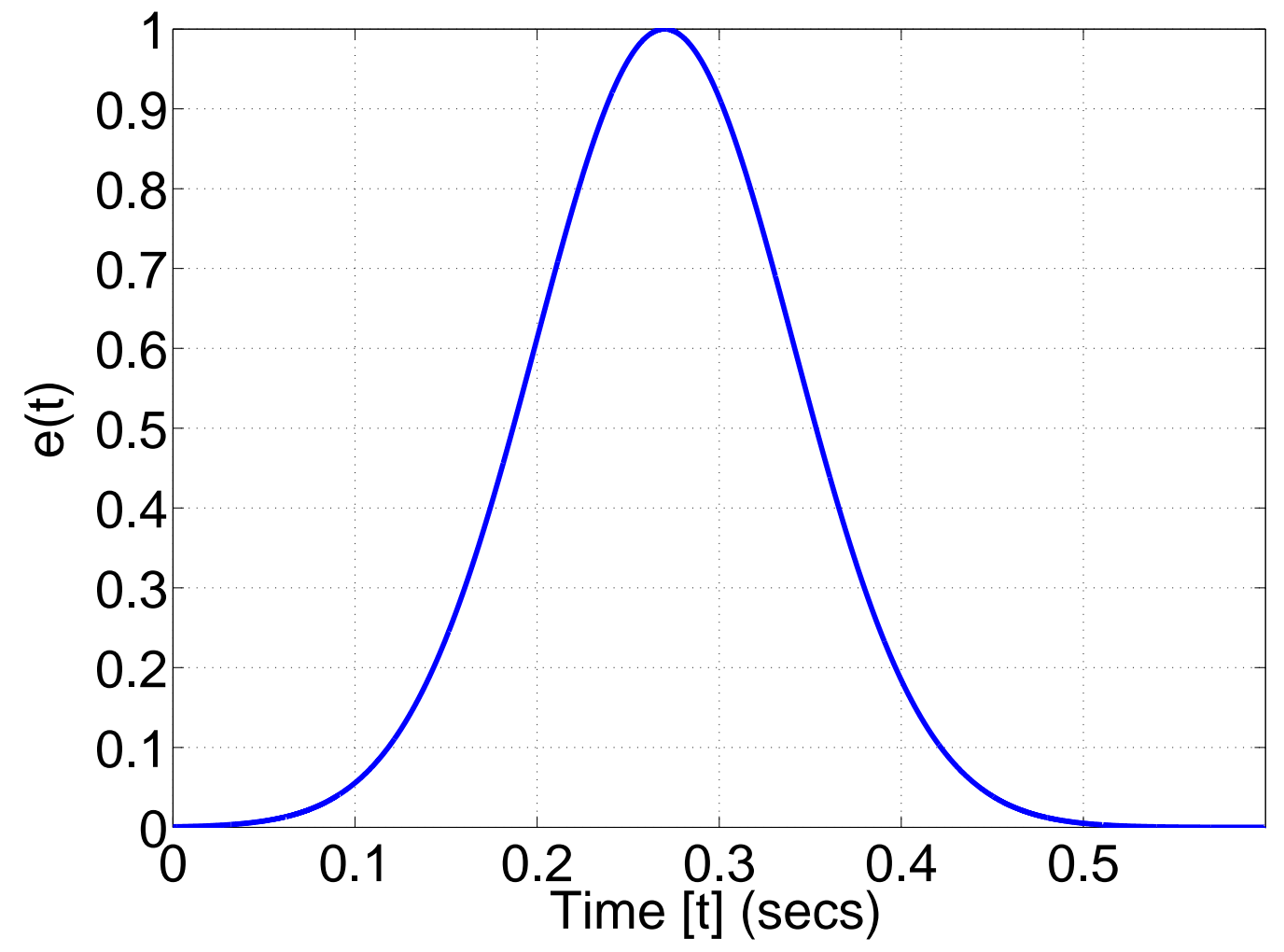

Figure 3: The model driver $(\mathrm{e}(\mathrm{t}))$. 

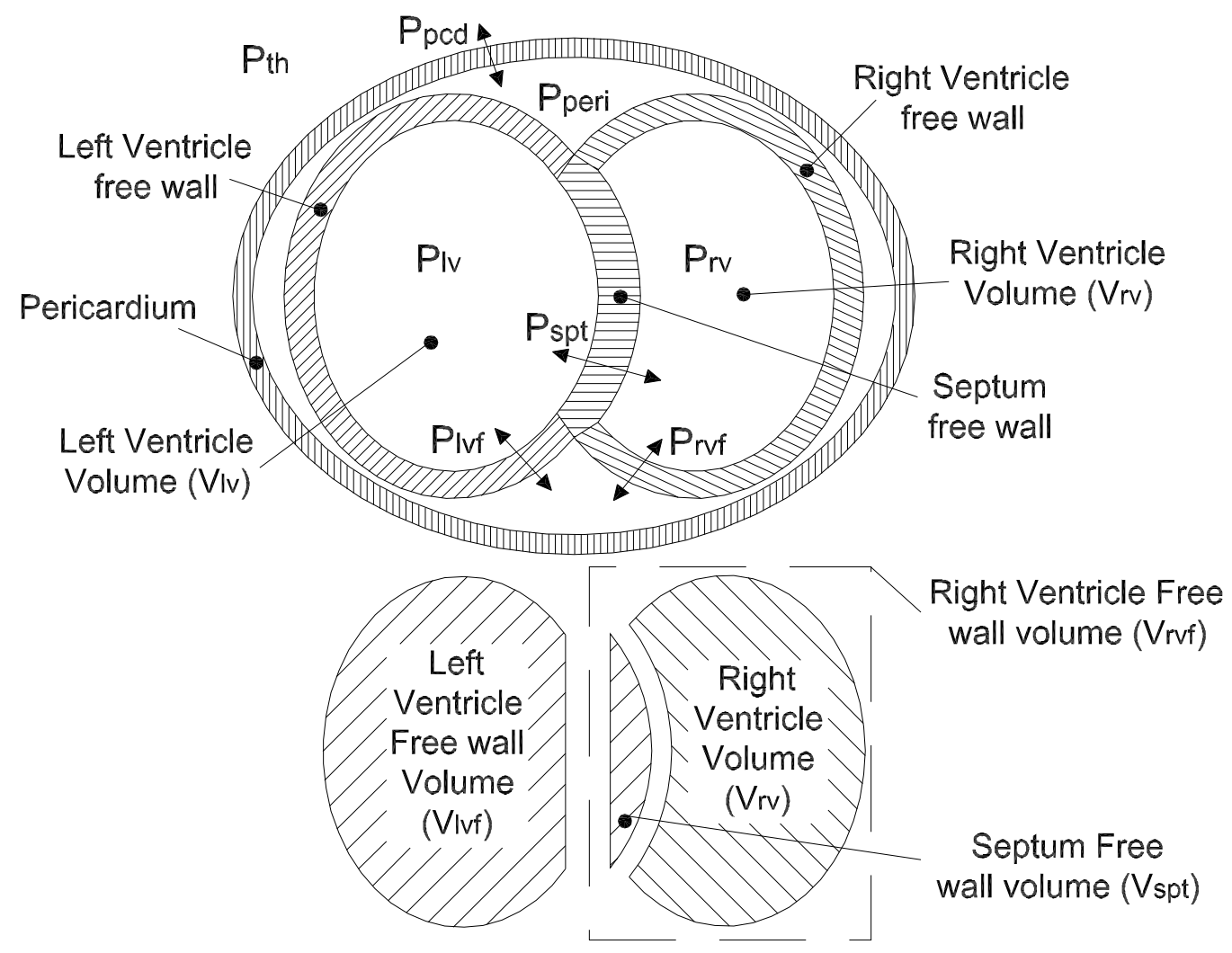

Figure 4: Pressure and volume definitions in the heart. 

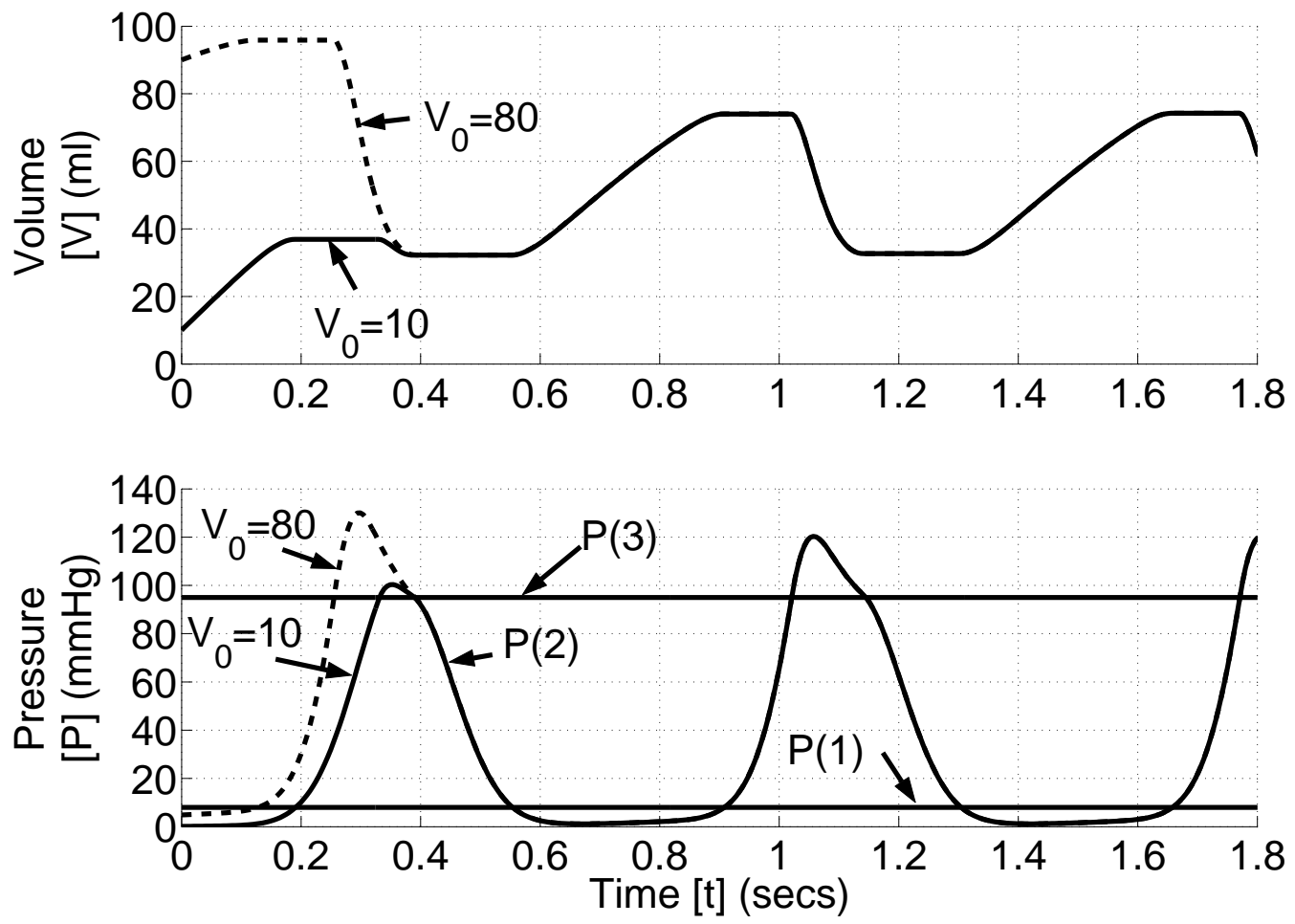

Figure 5: Response of the single chamber system for different initial conditions. 


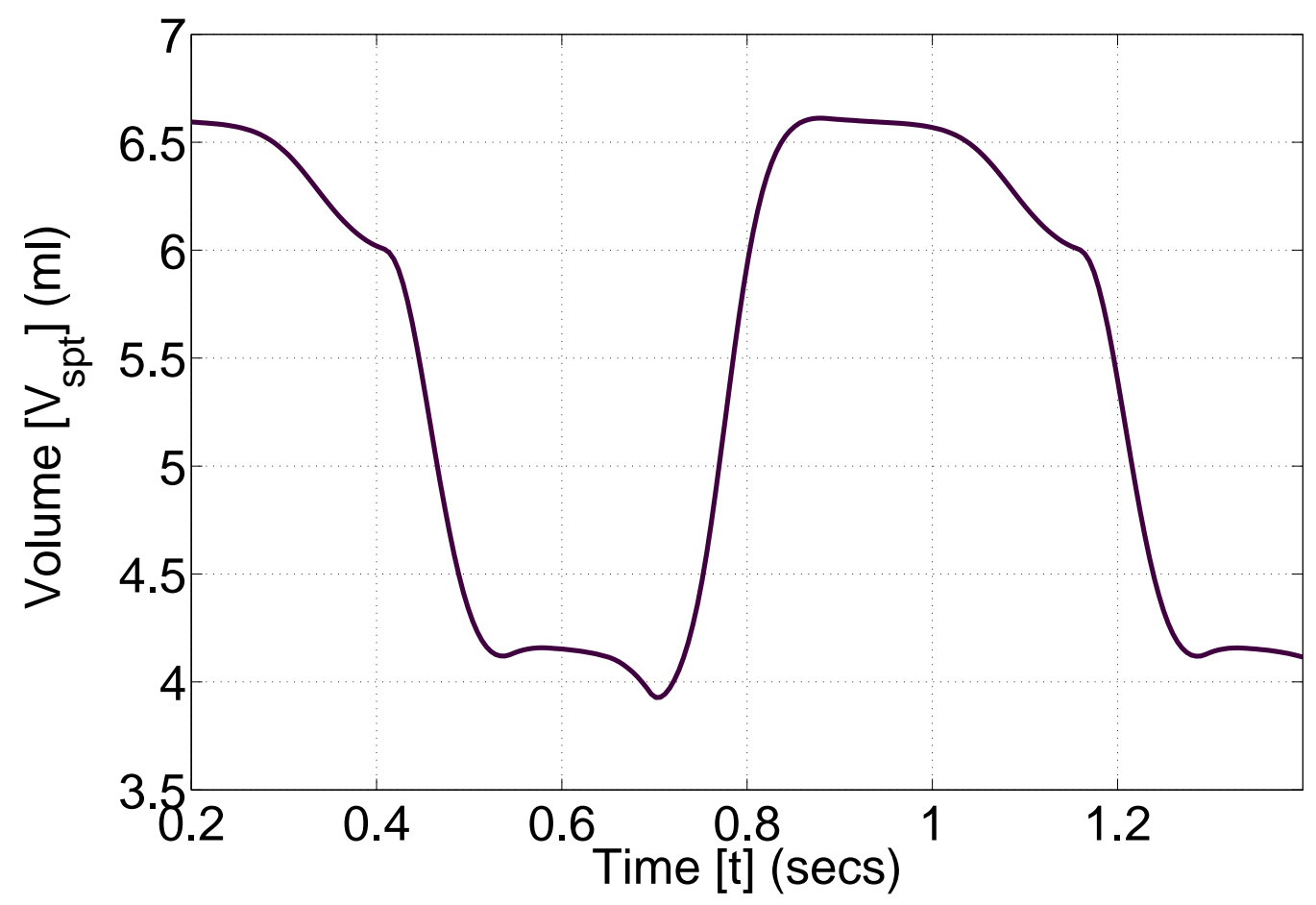

Figure 6: Graph showing changes in septum free wall displacement from the constant boundary condition, 2 chamber model. 

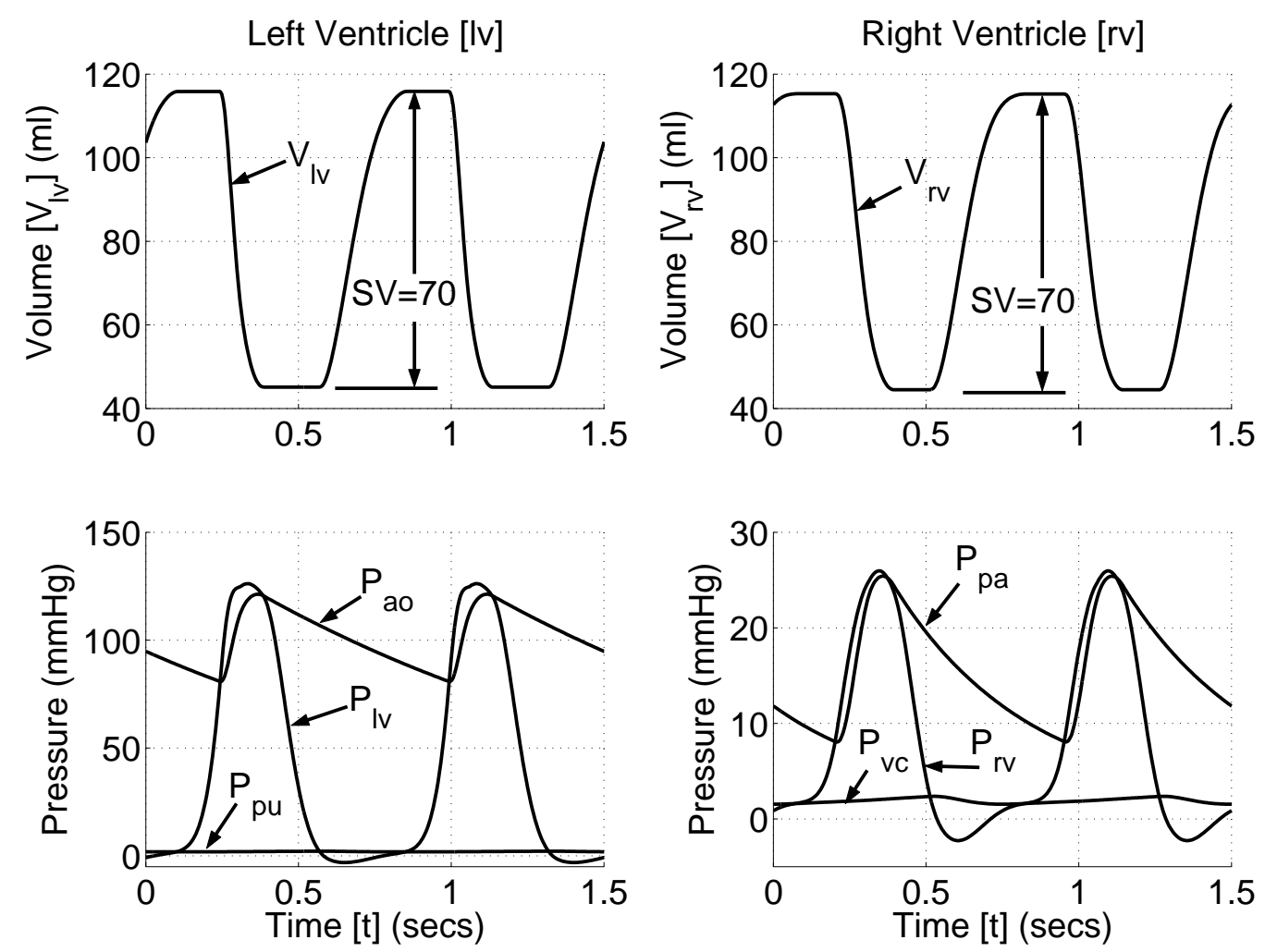

Figure 7: Simulation results from the closed loop model with inertia and ventricular interaction. 

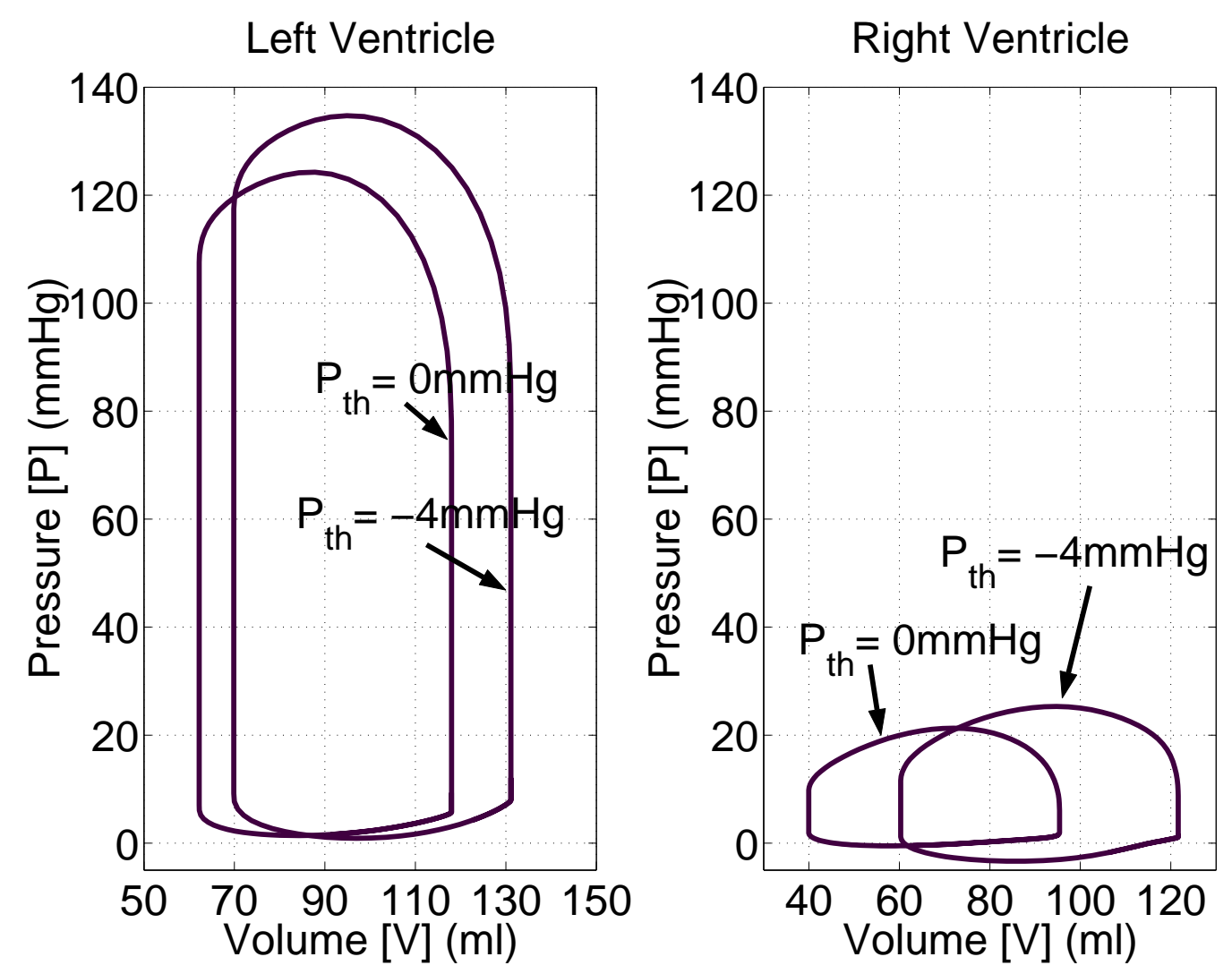

Figure 8: Change in stroke volume and PV diagram for a change in thoracic pressure. 

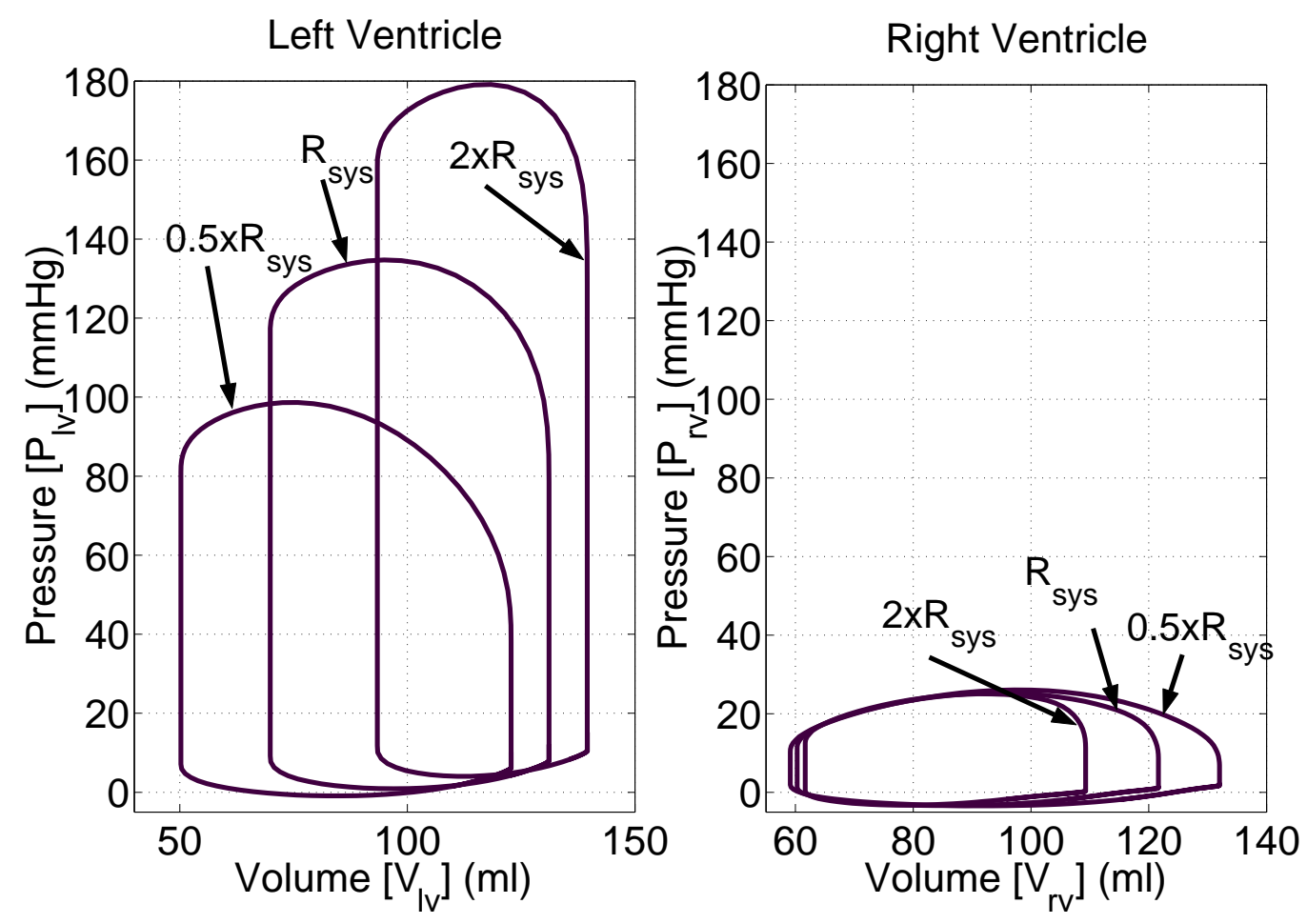

Figure 9: Simulation for changes in systemic resistance. 


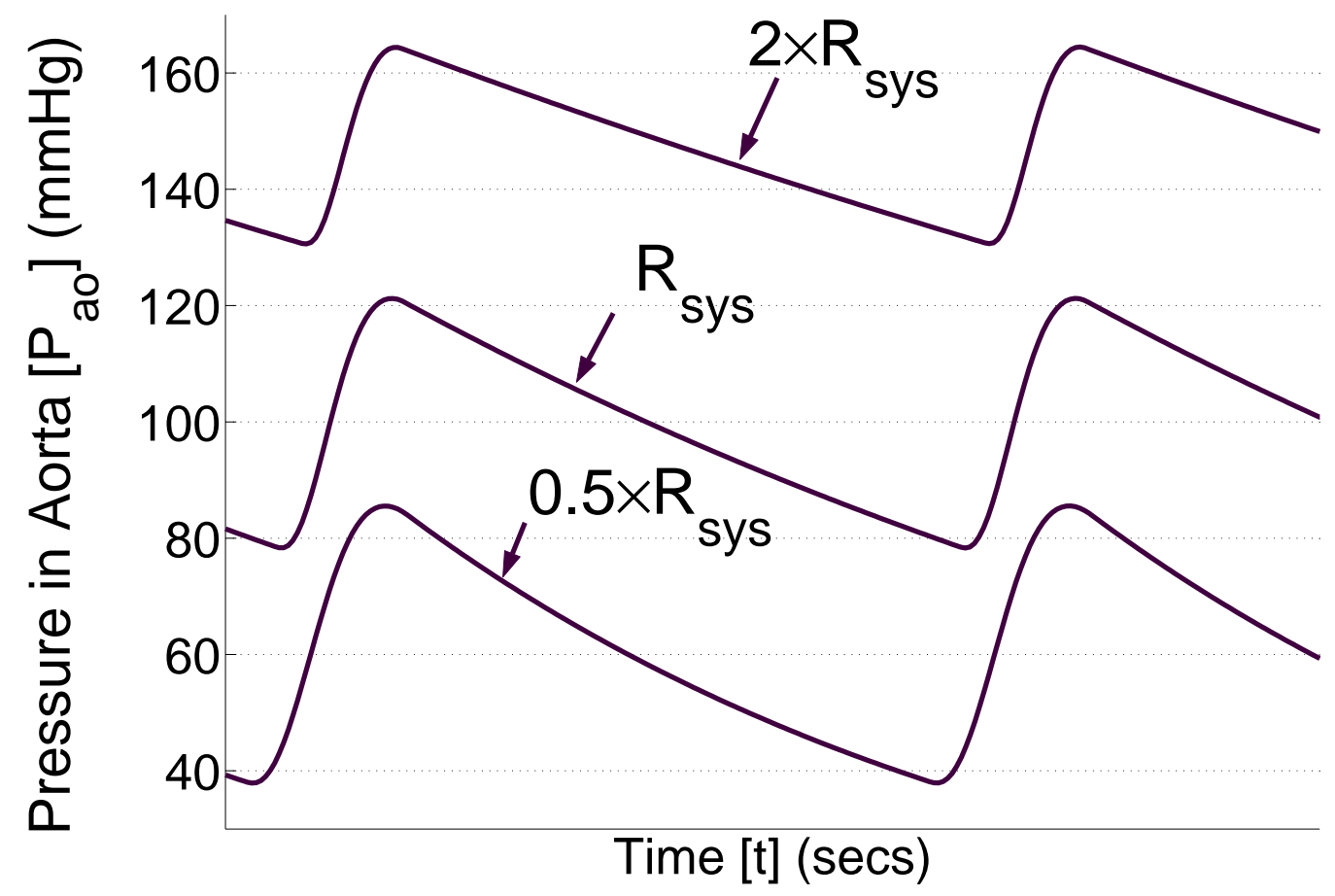

Figure 10: Response of aortic pressure to changes in systemic resistance. 

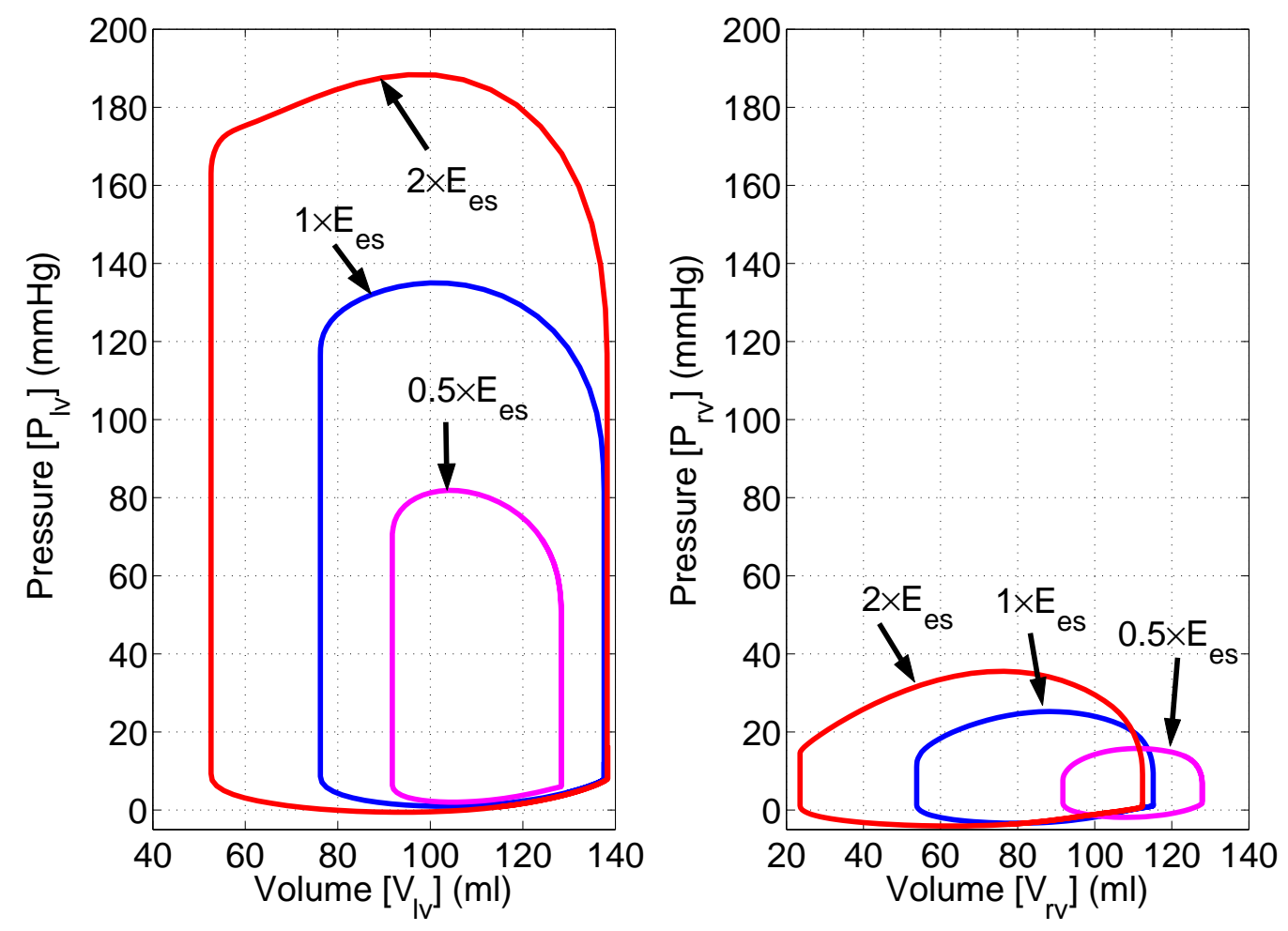

Figure 11: The effect of varying ventricular contractility. 


\begin{tabular}{|c|l|}
\hline Symbol & Description \\
\hline $\mathrm{V}_{\mathrm{lv}}$ & Left ventricle volume \\
$\mathrm{V}_{\mathrm{rv}}$ & Right ventricle volume \\
$\mathrm{V}_{\mathrm{lvf}}$ & Left ventricular free wall volume \\
$\mathrm{V}_{\mathrm{rvf}}$ & Right ventricular free wall volume \\
$\mathrm{V}_{\mathrm{spt}}$ & Septum free wall volume \\
$\mathrm{V}_{\mathrm{pcd}}$ & Pericardium volume \\
\hline $\mathrm{P}_{\mathrm{lv}}$ & Pressure in the left ventricle \\
$\mathrm{P}_{\mathrm{rv}}$ & Pressure in the right ventricle \\
$\mathrm{P}_{\mathrm{peri}}$ & Pressure in the pericardium \\
$\mathrm{P}_{\mathrm{th}}$ & Pressure in the thoracic cavity \\
$\mathrm{P}_{\mathrm{lvf}}$ & Pressure across the left ventricular free wall \\
$\mathrm{P}_{\mathrm{rvf}}$ & Pressure across the right ventricular free wall \\
$\mathrm{P}_{\mathrm{spt}}$ & Pressure across the septum free wall \\
$\mathrm{P}_{\mathrm{pcd}}$ & Pressure across the pericardium wall \\
\hline
\end{tabular}

Table 1: Volume and Pressure Variables. 